\title{
The DinJ/RelE Toxin-Antitoxin System Suppresses Bacterial Proliferation and Virulence of Xylella fastidiosa in Grapevine
}

\author{
Lindsey P. Burbank and Drake C. Stenger
}

United States Department of Agriculture-Agricultural Research Service, San Joaquin Valley Agricultural Sciences Center, Parlier, CA $93648-9757$. Accepted for publication 7 December 2016.

\begin{abstract}
Xylella fastidiosa, the causal agent of Pierce's disease of grapes, is a slow-growing, xylem-limited, bacterial pathogen. Disease progression is characterized by systemic spread of the bacterium through xylem vessel networks, causing leaf-scorching symptoms, senescence, and vine decline. It appears to be advantageous to this pathogen to avoid excessive blockage of xylem vessels, because living bacterial cells are generally found in plant tissue with low bacterial cell density and minimal scorching symptoms. The DinJ/RelE toxin-antitoxin system is characterized here for a role in controlling bacterial proliferation and population size during plant colonization. The DinJ/RelE locus is transcribed from two separate promoters, allowing for

coexpression of antitoxin DinJ with endoribonuclease toxin RelE, in addition to independent expression of RelE. The ratio of antitoxin/toxin expressed is dependent on bacterial growth conditions, with lower amounts of antitoxin present under conditions designed to mimic grapevine xylem sap. A knockout mutant of DinJ/RelE exhibits a hypervirulent phenotype, with higher bacterial populations and increased symptom development and plant decline. It is likely that DinJ/RelE acts to prevent excessive population growth, contributing to the ability of the pathogen to spread systemically without completely blocking the xylem vessels and increasing probability of acquisition by the insect vector.
\end{abstract}

Xylella fastidiosa, a phytopathogenic bacterium that causes Pierce's disease of grapevine as well as a number of other economically important plant diseases, encodes multiple toxin-antitoxin (TA) systems on its chromosome (Lee et al. 2014). X. fastidiosa is a slow-growing, fastidious organism that colonizes two very specific environmental niches: xylem tissue of the plant host and foregut of the insect vector (Chatterjee et al. 2008). Bacterial TA systems consist of a toxin which disrupts replication or cell function, and an inherently unstable antitoxin which specifically inhibits toxin activity. The presence of TA systems has been observed to contribute to development of persistent infections and long-term pathogen survival (Balaban et al. 2004; Fasani and Savageau 2015). The mechanism of TA system-facilitated persistence involves a reversible phenotypic change in bacterial cells to temporarily reduce growth and metabolic rates when the bacterial population is exposed to stress or starvation conditions. Thus, changes in the environmental conditions can dictate the rate of bacterial population expansion and invasion of the host (Lobato-Márquez et al. 2016). Although there has been some functional characterization of $X$. fastidiosa TA systems (Lee et al. 2012, 2014) and one (MqsR/MqsA) has been implicated in virulence in planta (Merfa et al. 2016), biological roles for the majority of $X$. fastidiosa TA systems have not been determined.

The chromosomal TA systems that have been identified in $X$. fastidiosa are classified as type II TA systems, in which the toxin protein is a ribonuclease that degrades single-stranded RNA with varying degrees of sequence specificity (Cook et al. 2013; Lee et al. 2012, 2014; Merfa et al. 2016; Syed and Lévesque 2012). In addition to a general function in control of bacterial growth and persistence through toxin activity, type II TA systems often have

Corresponding author: L. P. Burbank;

E-mail address: lindsey.burbank@ars.usda.gov

*The $\boldsymbol{e}$-Xtra logo stands for "electronic extra" and indicates that one supplementary figure and one supplementary table are published online.

This article is in the public domain and not copyrightable. It may be freely reprinted with customary crediting of the source. The American Phytopathological Society, 2017. regulatory roles in controlling complex cellular process such as biofilm formation and virulence (Kim et al. 2009; Merfa et al. 2016). TA systems have been identified in plant-pathogenic bacteria, particularly Xanthomonas spp.; and, in some cases, specific roles in virulence have been characterized (Martins et al. 2016; Triplett et al. 2016). The DinJ/RelE TA system in $X$. fastidiosa consists of an endoribonuclease toxin (RelE) and its cognate antitoxin (DinJ) which neutralizes toxin activity by direct binding (Lee et al. 2014). RelE digests single-stranded RNA with no apparent sequence specificity in vitro, resulting in reduction of bacterial growth in the absence of the antitoxin, DinJ (Lee et al. 2014). In this study, we explore the function of the $X$. fastisiosa DinJ/RelE TA system in relation to control of bacterial population growth and virulence in the plant.

\section{MATERIALS AND METHODS}

Bacterial strains and culture conditions. Escherichia coli strain Top10 (Thermo Fisher Scientific) was used as a cloning host. E. coli was grown in Luria-Bertani medium at $37^{\circ} \mathrm{C}$ unless otherwise indicated, with the addition of antibiotics when necessary. For E. coli, antibiotics were used at the following concentrations: spectinomycin at $100 \mu \mathrm{g} / \mathrm{ml}$, chloramphenicol at $35 \mu \mathrm{g} / \mathrm{ml}$, and gentamycin at $10 \mu \mathrm{g} / \mathrm{ml}$. $X$. fastidiosa wild-type Stag's Leap (GenBank accession number LSMJ00000000.1) (Chen et al. 2016) and mutant derivatives were grown in Pierce's disease medium (PD3) (Davis et al. 1981) or xylem chemistry based defined medium (Chard2) (Leite et al. 2004) at $28^{\circ} \mathrm{C}$. For $X$. fastidiosa strains, PD3 was supplemented when necessary with gentamycin at $5 \mu \mathrm{g} / \mathrm{ml}$ or chloramphenicol at $5 \mu \mathrm{g} / \mathrm{ml}$. Details and sources of bacterial strains used in this study are presented in Table 1.

Knockout mutant construction. Double mutant $\Delta$ dinJrelE, complemented strain $\Delta$ dinJrelE/dinJrelE ${ }^{+}$, and empty-vector control $\Delta$ dinJrelE pBBRpemIK were created in a previous study (Burbank and Stenger 2016a). The single mutant in relE (PD1100) was created by replacing the open reading frame (ORF) with an antibiotic resistance marker gene (chloramphenicol acetyl transferase) using homologous recombination, as previously described (Burbank and Stenger 2016b). Briefly, 1-kbp DNA fragments upstream and downstream of the relE ORF were polymerase chain reaction (PCR) amplified using Stag's Leap genomic DNA as template (primer pairs relEupfwd/relEuprev 
and relEdnfwd/relEdnrev). Primer sets were designed to have 15 nucleotides homologous to the chloramphenicol resistance marker, which was amplified separately from the Tn5 HyperMu transposon (Epicentre) with primers Chlfwd and Chlrev. Three DNA fragments (upstream and downstream flanking regions and chloramphenicol resistance marker) were annealed and assembled using overlap extension PCR (Heckman and Pease 2007). The combined PCR product of approximately 3-kbp was gel purified and cloned into vector pCR8/GW/TOPO, following the manufacturer's instructions, to create plasmid construct pCR8-relE:chl. This plasmid was transformed into $X$. fastidiosa by electroporation, as previously described (Guilhabert et al. 2001). Homologous recombination and gene replacement were selected for by plating transformants on PD3 with chloramphenicol. Colonies resistant to chloramphenicol were screened by colony PCR using primer set RST31/RST33 (Minsavage et al. 1994) (X. fastidiosaspecific primers) and gene-specific primers (relEfwd/relErev). The correct mutant $(\Delta r e l E)$ was further confirmed by cloning and sequencing the site of recombination (Burbank and Stenger 2016a). Complete sequences of all PCR primers used in this study are presented in Supplementary Table S1.

Verification of dinJ-relE operon transcripts. Total RNA was extracted from $X$. fastidiosa cultures after 5 days of growth on PD3 plates using the hot phenol extraction method described by Jahn et al. (2008). RNA samples were treated with Ambion Turbo Dnase (Thermo Fisher Scientific) and RNA integrity was evaluated by agarose gel electrophoresis. Total RNA $(1 \mu \mathrm{g})$ was used as template for $5^{\prime}$ rapid amplification of cDNA ends (RACE) (Scotto-Lavino et al. 2007) using the 5' RACE System kit (Thermo Fisher Scientific), with primer relERACE1 for initiation of reverse transcription. 5' RACE products were PCR amplified using primers relERACE2 and Abridged Anchor Primer (provided as part of the kit). PCR products were gel purified and cloned into $\mathrm{pCR} 8 / \mathrm{GW} / \mathrm{TOPO}$ for sequencing.

Gene expression analysis. Cells were harvested from PD3 plates after 1 week of growth and resuspended in liquid PD3 or Chard 2 to a cell optical density at $600 \mathrm{~nm}\left(\mathrm{OD}_{600}\right)$ of 0.25 . Cell suspensions $(1 \mathrm{ml})$ were diluted in $25 \mathrm{ml}$ of liquid media (PD3 or Chard2). Total RNA was extracted from cells after 5 days of growth in liquid media using a hot phenol extraction method (Jahn et al. 2008). RNA samples were treated with Turbo Dnase and RNA integrity was evaluated by agarose gel electrophoresis. Total RNA $(1 \mu \mathrm{g})$ was used to make cDNA using the iScript cDNA synthesis kit (Bio-Rad). Quantitative PCR (qPCR) was performed with primers relEqPCRfwd/relEqPCRrev ( relE), dinJqPCRfwd/dinJqPCRrev $(d i n J)$, or dnaQfwd/dnaQrev (reference gene $d n a Q$ ) and Applied
Biosystems Fast SYBR Green Master Mix. SYBR green fluorescence detection was performed on the Applied Biosystems StepOne Plus instrument and expression analysis was completed using the associated StepOne Plus software package. Individual primer sets were validated for PCR efficiency and specificity by standard curve and melt curve analysis. DnaQ (DNA polymerase subunit) has previously been validated for use as a reference gene in $X$. fastidiosa (Burbank and Stenger 2016a; Zaini et al. 2008), with stable expression observed across different treatments. Relative normalized gene expression was calculated based on the $2^{-\Delta \Delta \mathrm{Ct}}$ method (Livak and Schmittgen 2001). Gene expression analysis was performed on at least three independent biological replicates for each strain, with three PCR replicates for each biological replicate.

Reverse transcription. Reverse transcription was performed using the iScript Select cDNA synthesis kit (Bio-Rad), with genespecific primers relERACE1 and dinJrev to target the dinJ and relE transcripts. Total RNA $(1 \mu \mathrm{g})$ from cells grown in either PD3 or Chard 2 and extracted following the same protocol described for qPCR was used as template for reverse transcription. cDNA was amplified with 20 cycles of PCR using primers relEfwd/relERACE1 for $r e l E$ transcripts and dinJfwd/dinJrev for dinJ transcripts.

relE expression from individual promoters. For expression analysis of relE with individual promoters $\mathrm{P} 1$ and $\mathrm{P} 2$, the relE mutant was complemented with the relE ORF fused to either P1 ( $\Delta$ relE/relE-P1) or P2 ( $\Delta$ relE/relE-P2) by chromosomal insertion, as previously described (Matsumoto et al. 2009). Strain $\Delta$ relE/relE$P 1$ was constructed by PCR amplifying dinJ-relE using primers dinJrelEORFfwd/dinJrelEORFrev and cloning into vector pCR8/ GW/TOPO to create plasmid pCR8-dinJrelE. The dinJ ORF (which contains promoter $\mathrm{P} 2$ ) was then removed using the Q5 site-directed mutagenesis kit (New England Biolabs) to create plasmid pCR8relE-P1. Mutagenesis primers (pDinJrelErev/relEORFfwd) were designed to remove the dinJ ORF, leaving relE in frame with promoter $\mathrm{P} 1$. Strain $\Delta$ relE/relE-P2 was constructed by PCR amplifying the relE ORF along with $150 \mathrm{bp}$ upstream of the transcription start (determined by $5^{\prime}$ RACE, as described above) using primers pRelEfwd/RelErev and cloned into pCR8/GW/TOPO following the manufacturer's instructions to create plasmid pCR8-relE-P2. Plasmid constructs pCR8-relE-P1 and pCR8-relE-P2 were recombined with Gateway cloning-compatible chromosomal insertion vector pAX1-GW (Burbank et al. 2015; Matsumoto et al. 2009) using LR clonase enzyme mix (Thermo Fisher Scientific). New plasmid constructs pAX1-relE-P1 and pAX1-relE-P2 were transformed into $X$. fastidios $a$ by electroporation and homologous recombination was confirmed by cloning

TABLE 1. Bacterial strains and plasmids used in this study

\begin{tabular}{|c|c|c|}
\hline Strains & Characteristics $^{\mathrm{a}}$ & Source \\
\hline \multicolumn{3}{|l|}{ Xylella fastisioda subsp. } \\
\hline$\Delta$ dinJrelE & Stag's Leap knockout mutant in toxin-antitoxin system dinJ/relE, $\mathrm{Cm}^{\mathrm{R}}$ & Burbank and Stenger 2016a \\
\hline$\Delta$ dinJrelE/dinJrelE ${ }^{+}$ & $\Delta$ dinJ/relE, complemented using plasmid pBBR5pemIK, $\mathrm{Cm}^{\mathrm{R}}, \mathrm{Gm}^{\mathrm{R}}$ & Burbank and Stenger 2016a \\
\hline$\Delta$ dinJrelE pBBR5pemIK & Empty-vector control for $\Delta$ dinJ/relE plasmid complemented strain, $\mathrm{Cm}^{\mathrm{R}}, \mathrm{Gm}^{\mathrm{R}}$ & Burbank and Stenger 2016a \\
\hline$\Delta r e l E$ & Knockout mutant in $r e l E, \mathrm{Cm}^{\mathrm{R}}$ & This study \\
\hline$\Delta r e l E / r e l E-P 1$ & $\Delta r e l E$ knockout mutant complemented with relE ORF and promoter $\mathrm{P} 1$ & This study \\
\hline$\Delta r e l E / r e l E-P 2$ & $\Delta$ relE knockout mutant complemented with relE ORF and promoter P2 & This study \\
\hline Escherichia coli Top 10 & $\begin{array}{l}\text { Cloning host, F- mcrA } \Delta(m r r-h s d \mathrm{RMS}-m c r \mathrm{BC}), \Phi 80 \text { lac } \mathrm{Z} \Delta \mathrm{M} 15, \Delta l a c \mathrm{X} 74, \text { rec } \mathrm{A} 1 \text {, } \\
\text { araD139, } \Delta(\text { araleu }) 7697, \text { gal } \mathrm{U}, \text { gal } \mathrm{K}, \operatorname{rps} \mathrm{L},\left(\mathrm{Str}^{\mathrm{R}}\right), \text { end } \mathrm{A} 1, \text { nup } \mathrm{G}\end{array}$ & Thermo Fisher Scientific \\
\hline \multicolumn{3}{|c|}{ (2) } \\
\hline pCR8/GW/TOPO & TOPO cloning vector, $\mathrm{Sp}^{\mathrm{R}}$, att sites for Gateway cloning & Thermo Fisher Scientific \\
\hline pBBR5pemIK & Broad host range vector containing pemI/pemK stability factor, $\mathrm{Gm}^{\mathrm{R}}$ & Burbank and Stenger 2016a \\
\hline pBBR5pemIK-dinJrelE & Complementation construct for $\Delta$ dinJrelE knockout mutant & Burbank and Stenger 2016a \\
\hline pCR8-relE-P1 & pCR8/GW/TOPO containing relE ORF with promoter P1 & This study \\
\hline pCR8-relE-P2 & pCR8/GW/TOPO containing relE ORF with promoter P2 & This study \\
\hline $\mathrm{pAX} 1-\mathrm{GW}$ & Gateway cloning compatible chromosomal insertion vector for $X$. fastidiosa & $\begin{array}{l}\text { Burbank and Stenger 2016b, } \\
\text { Matsumoto et al. } 2009\end{array}$ \\
\hline pAX1-relE-P1 & pAX1-GW carrying relE ORF with promoter P1 & This study \\
\hline pAX1-relE-P2 & pAX1-GW carrying relE ORF with promoter P1 & This study \\
\hline
\end{tabular}

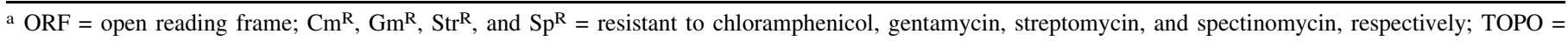
topoisomerase based cloning. 
and sequencing the insertion site. Gene expression analysis was performed with strains $\Delta r e l E / r e l E-P 1$ and $\Delta r e l E / r e l E-P 2$ following growth in PD3 or Chard2, as described above.

Cell viability assays. Wild-type Stag's Leap and mutant strain $\Delta$ dinJrelE along with complemented strain $\Delta$ dinJrelE/dinJrelE ${ }^{+}$ and empty-vector control strain $\Delta$ dinJrelE-pBBR5pemIK were grown for 7, 10, or 14 days on PD3 plates. Cells were harvested from individual plates in $4 \mathrm{ml}$ of phosphate-buffered saline $(1 \times \mathrm{PBS}) \mathrm{pH} 7.4$. Aliquots $(1 \mathrm{ml})$ from each sample were used for fluorescent viability assays and for total cell quantification by qPCR. To determine total cell quantity, genomic DNA was extracted using a DNeasy Blood and Tissue Kit (Qiagen) and quantified by qPCR with Applied Biosystems Fast SYBR Green master mix (Thermo Fisher Scientific) and $X$. fastidiosa-specific primers Xf145-60fwd/Xf145-60rev. Cell quantity was determined based on a DNA standard curve made from $X$. fastidiosa cells diluted to known concentrations (CFU per milliliter). Cell viability was determined with Alamar Blue (Thermo Fisher Scientific) fluorescent cell viability reagent following the manufacturer's protocol. Briefly, 1-ml aliquots of cells were harvested by centrifugation and resuspended in $90 \mu \mathrm{l}$ of $1 \times$ PBS. Cells were combined with $10 \mu \mathrm{l}$ of Alamar Blue reagent in solid black 96-well plates (Corning Inc.). Plates were then wrapped in aluminum foil and incubated for $2 \mathrm{~h}$ at $28^{\circ} \mathrm{C}$. After incubation, fluorescence was read using an Infinite M1000 Pro plate reader (Tecan Systems Inc.) at 570-nm excitation and 585-nm emission wavelengths. A standard curve showing correlation between Alamar Blue fluorescence and proportion of live cells for $X$. fastidiosa is shown in Supplementary Figure S1.

Virulence assays. Susceptible grapevines (Vitis vinifera 'Chardonnay') were needle inoculated with wild-type Stag's Leap, $\Delta$ dinJrelE, complemented mutant $\Delta$ dinJrelE pBBR5pemIK-dinJrelE (Burbank and Stenger 2016a), and empty-vector control strain $\triangle$ dinJrelE pBBR5pemIK. Plants were observed for development of Pierce's disease symptoms over the course of 12 weeks. Plants (20 plants/treatment) were assigned a disease rating between 0 and 5 once per week based on the system of Roper et al. (2007). Bacterial quantity was determined by qPCR at 10 weeks postinoculation, as previously described (Burbank and Stenger 2016b). Primers Xf14560fwd/Xf145-60rev (Ledbetter and Rogers 2009) were used for qPCR with Applied Biosystems SYBR Green Master Mix (Thermo Fisher Scientific). Fluorescent quantitation and data analysis were carried out on a StepOne Plus instrument (Applied Biosystems), with $X$. fastidiosa quantity determined based on a standard curve of wild-type Stag's Leap genomic DNA. Statistical significance was evaluated using one-way analysis of variance followed by a TukeyKramer multiple comparison test $(\alpha=0.05)$. All statistical analyses were conducted using the Origin 2016 statistical analysis software package (OriginLabs).

\section{RESULTS}

$\Delta$ dinJrelE operon contains two promoters. TA system DinJ/RelE is encoded by overlapping dinJ and relE ORF in the same

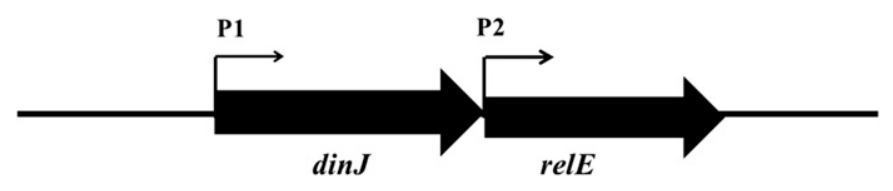

P1

$\mathbf{P 2}$

Fig. 1. Operon structure of dinJ-relE locus. Gene annotation for dinJ-relE (locus tag PD1099/PD1100) was obtained from the MicrobesOnline database (Dehal et al. 2009). Narrow black arrows indicate transcript lengths and bent arrows indicate promoter location. Transcript lengths were determined by $5^{\prime}$ rapid amplification of cDNA ends (RACE) analysis from total RNA extracted from Xylella fastidiosa cultures after 5 days of growth on PD3 plates. 5' RACE was performed with $1 \mu \mathrm{g}$ of total RNA using 5' RACE System Kit (Thermo Fisher Scientific). orientation (Fig. 1). 5' RACE analysis to identify the transcription start site of relE produced two distinct products, one of $282 \mathrm{bp}$ (relE) and one of approximately $500 \mathrm{bp}$, corresponding to the size of dinJrelE together. Sequences of the two 5' RACE products confirmed identity of the transcripts and transcription initiation sites. Identification of two transcripts suggests the presence of two promoters, one located upstream of dinJ for cotranscription of $\operatorname{dinJ}$ and relE (P1) and one located internally for transcription of relE alone (P2) (Fig. 1).

Transcriptional expression levels of $\mathrm{dinJ}$ and relE are dependent on growth conditions. Transcriptional expression of dinJ and relE in wild-type Stag's Leap were compared using qPCR during growth in rich medium (PD3) and a defined medium engineered to mimic grapevine xylem sap (Chard2) (Leite et al. 2004) (Fig. 2A). In Chard 2 medium, dinJ and relE were expressed at the same level. However, in PD3 medium, dinJ (antitoxin) was

A

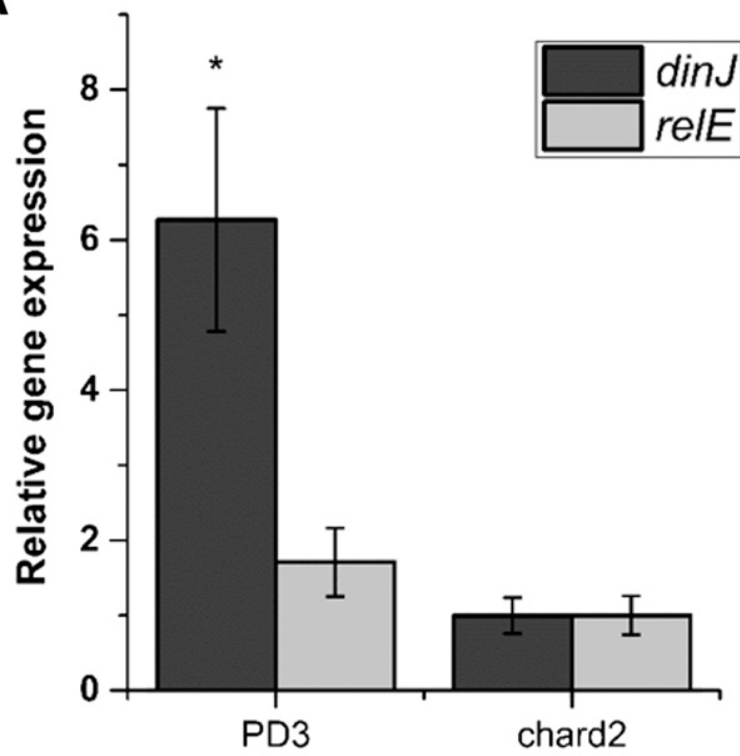

AT:T ratio $\quad 3.7: 1 \quad 1: 1$

B

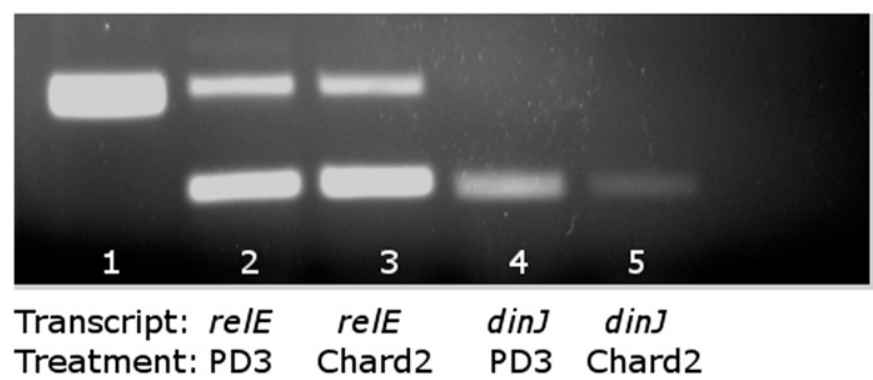

Fig. 2. Relative expression of $\operatorname{din} J$ and relE is dependent on growth conditions. A, Gene expression of $\operatorname{din} J$ and relE was compared for wild-type Stag's Leap using quantitative polymerase chain reaction (PCR) with $d n a Q$ as a reference gene. Prior to RNA extraction and cDNA synthesis, cells were grown in $25 \mathrm{ml}$ of liquid media (PD3 or Chard2). Total RNA ( $1 \mu \mathrm{g})$ was used to make cDNA using the iScript cDNA synthesis kit (Bio-Rad). Gene expression analysis was performed on at least three independent biological replicates for each strain, with three PCR replicates for each biological replicate. An asterisk (*) indicates samples that are significantly different from the relE transcript level under the same conditions based on a Student's $t$ test using $P \leq 0.01$. The antitoxin/toxin (AT:T) ratio was calculated as relative antitoxin $(\operatorname{din} J)$ expression divided by relative toxin (relE) expression. B, Reverse transcription was performed using the iScript Select cDNA synthesis kit with gene-specific primers to show transcript lengths from cells grown in PD3 and Chard2. Lane 1 = size marker of 500 $\mathrm{bp}$, lane $2=$ relE transcripts from cells grown in $\mathrm{PD} 3$, lane $3=$ relE transcript from cells grown in Chard2, lane $4=\operatorname{din} J$ transcript from cells grown in PD3, and lane $5=\operatorname{din} J$ transcript from cells grown in Chard2. 
expressed at a sixfold higher level compared with relE (Fig. 2A). The amount of dinJ expressed in PD3 was significantly different from the amount of relE based on a Student's $t$ test using $P \leq 0.01$. There was no significant difference between relE and $\operatorname{dinJ}$ expression at the transcriptional level in Chard2. Products of reverse transcription following growth in either PD3 or Chard2 (Fig. 2B) confirm the presence of both individual transcripts dinJ and relE, and the combined full-length transcript of both genes. Expression of relE from either promoter $\mathrm{P} 1$ or $\mathrm{P} 2$ was then characterized using strains $\Delta$ relE/relE-Pland $\Delta$ relE/relE-P2, which were designed to express relE from the individual promoters in the $\Delta r e l E$ mutant background (Fig. 3). In PD3, transcriptional expression of relE from P1 was approximately twofold higher than expression from promoter P2. This difference was significant based on a Student's $t$ test using $P \leq$ 0.01 . For cells grown in Chard2, there was no significant difference between relE expression from P1 and P2 (Fig. 3).

TA system DinJ/RelE does not affect long-term survival of $X$. fastidiosa in vitro. Cell viability of wild-type Stag's Leap, $\Delta$ dinJrelE, $\Delta$ dinJrelE/dinJrelE ${ }^{+}$, and empty-vector control was determined over the course of 7 and 14 days of growth on PD3 plates using the Alamar Blue fluorescent cell viability assay (Fig. 4). The Alamar Blue reagent is a cell-permeable dye which produces measurable fluorescence upon exposure to the reducing environment of living bacterial cells (Shiloh et al. 1997). There is a linear correlation between amount of fluorescence produced after a given period of time (normalized to total number of cells) and the proportion of living cells present. This method of viable cell quantitation was used to reduce experimental variability inherent in colony counting assays. Proportion of viable cells out of total cells (determined by qPCR) was higher in the $\Delta$ dinJrelE mutant at 7 days compared with the wild type, consistent with what has previously been described (Fig. 4A) (Lee et al. 2014). This difference was significant based on one-way analysis of variance followed by a Tukey-Kramer multiple comparison test $(\alpha=0.05)$. However, after 14 days of growth, there was no significant difference in cell viability between any of the strains tested (Fig. 4B).

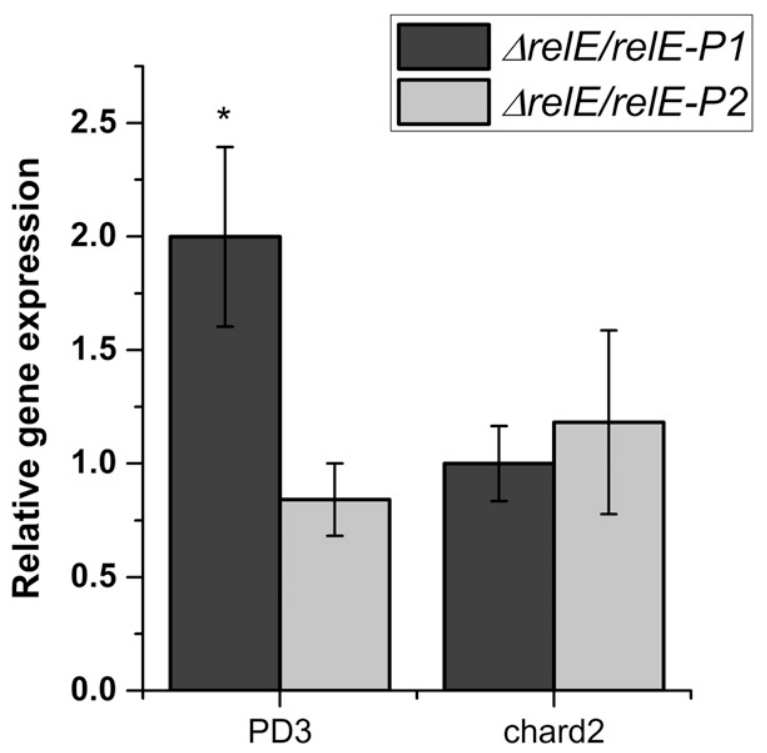

Fig. 3. Promoter $P 1$ has greater activity in rich medium. Gene expression of relE was compared in $\Delta r e l E$ mutant strains complemented with the relE open reading frame fused to one of the two promoters (P1 and P2) using quantitative polymerase chain reaction (PCR) with $d n a Q$ as a reference gene. Prior to RNA extraction and cDNA synthesis, cells were grown in $25 \mathrm{ml}$ of liquid medium (PD3 or Chard2). Total RNA (1 $\mu \mathrm{g})$ was used to make cDNA using the iScript cDNA synthesis kit (Bio-Rad). Gene expression analysis was performed on at least three independent biological replicates for each strain, with three PCR replicates for each biological replicate. An asterisk (*) indicates samples that are significantly different from the rest based on a Student's $t$ test using $P \leq 0.01$.
$\Delta$ dinJrelE mutant is hyper-virulent in planta. Bacterial quantity in infected plants as determined by qPCR was greater in $\Delta$ dinJrelE-inoculated plants after 12 weeks postinoculation compared with wild-type Stag's Leap-inoculated plants (Fig. 5A). In addition, visible symptoms were increased in grapevines inoculated with $\Delta$ dinJrelE (Fig. 5B and C). After 12 weeks postinoculation, the mean disease rating for $\Delta$ dinJrelE inoculated plants was $3.5 \pm 0.6$ compared with wild-type inoculated plants, which had a mean disease score of $1.9 \pm 1$ (Fig. 5B). Complemented strain $\Delta$ dinJrelE/ $\operatorname{dinJrelE} E^{+}$, which carries a wild-type copy of the $\operatorname{dinJ}$-relE operon on stable, broad-host-range plasmid pBBR5pemIK, was not

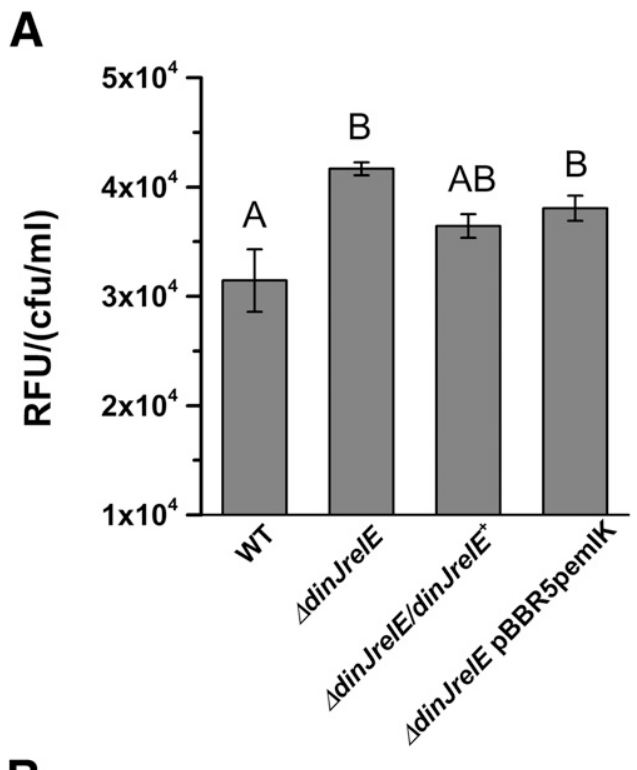

B

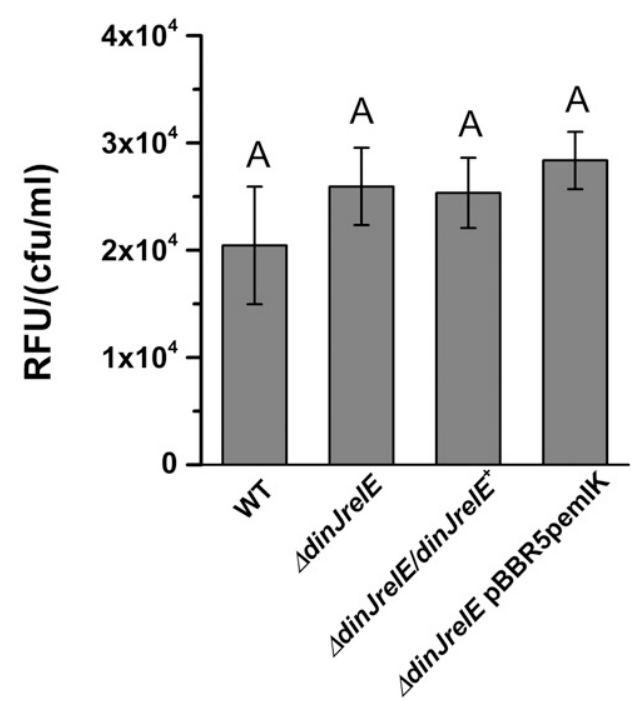

Fig. 4. Increased cell viability in $\Delta$ dinJrelE during in vitro growth does not negatively affect long-term survival. Cell viability was determined after $\mathbf{A}, 7$ and $\mathbf{B}, 14$ days of growth on PD3 plates using Alamar Blue fluorescent cell viability reagent. Viability represented in relative fluorescence units (RFU) was normalized to total cells (live and dead) as determined by quantitative polymerase chain reaction using Xylella fastidiosa-specific primers Xf14560fwd/Xf145-60rev. WT = wild type. Total cell quantity was determined based on a DNA standard curve made from $X$. fastidiosa cells diluted to known concentrations $(\mathrm{CFU} / \mathrm{ml})$. Alamar Blue fluorescence was read using an Infinite M1000 Pro plate reader (Tecan Systems Inc., San Jose, CA) at 570-nm excitation and 585-nm emission wavelengths. Standard curve showing correlation between Alamar Blue fluorescence and proportion of live cells for $X$. fastidiosa is shown in Supplementary Figure S1. Bars with different letters are significantly different based on one-way analysis of variance followed by Tukey-Kramer means comparison test $(\alpha=0.05)$. 
significantly different from the wild type in either bacterial quantity or symptom development (Fig. 5A, B, and C). Empty-vector control strain $\triangle$ dinJrelE $\mathrm{pBBR} 5 \mathrm{pemIK}$ was not significantly different from the mutant $\Delta$ dinJrelE. Significance of results was determined using one-way analysis of variance followed by a Tukey-Kramer multiple comparison test $(\alpha=0.05)$.

\section{DISCUSSION}

TA system control of bacterial population growth by bacteriostatic activity of the toxin has been well characterized in multiple bacterial species (Korch et al. 2009; Pedersen et al. 2002; Yamaguchi and Inouye 2011). For X. fastidiosa, pathogen transmission and host colonization are dependent on bacterial population behaviors such as biofilm formation and cell-density-dependent production of exopolysaccharides, adhesins, and plant cell-walldegrading enzymes (Chatterjee et al. 2010; Killiny and Almeida 2009a,b; Roper et al. 2007; Wang et al. 2012). Much of this behavior is attributed to production of a diffusible signal factor which regulates phenotypic changes in a cell-density-dependent manner, allowing the pathogen to move from one xylem vessel to another and systemically colonize the plant (Newman et al. 2004). In the plant, $X$. fastidiosa is generally present as dispersed populations of low cell density (Newman et al. 2003). Excessive bacterial growth and complete blockage of xylem vessels may actually be detrimental to long-term survival of the pathogen and probability of acquisition by the insect vector due to feeding preference for asymptomatic plants (Daugherty et al. 2011).
A

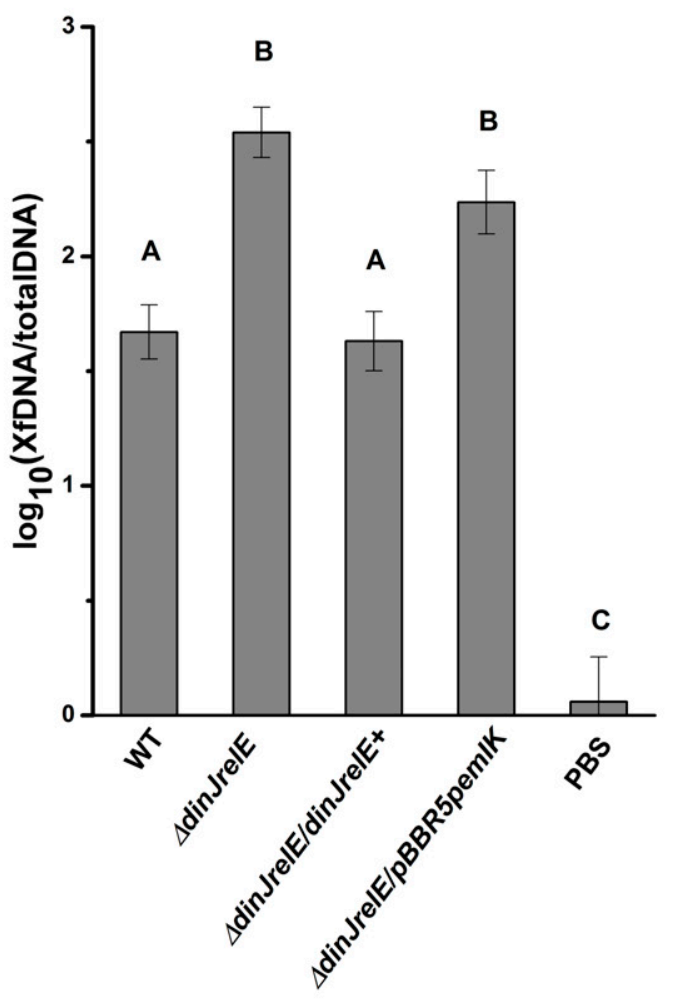

B

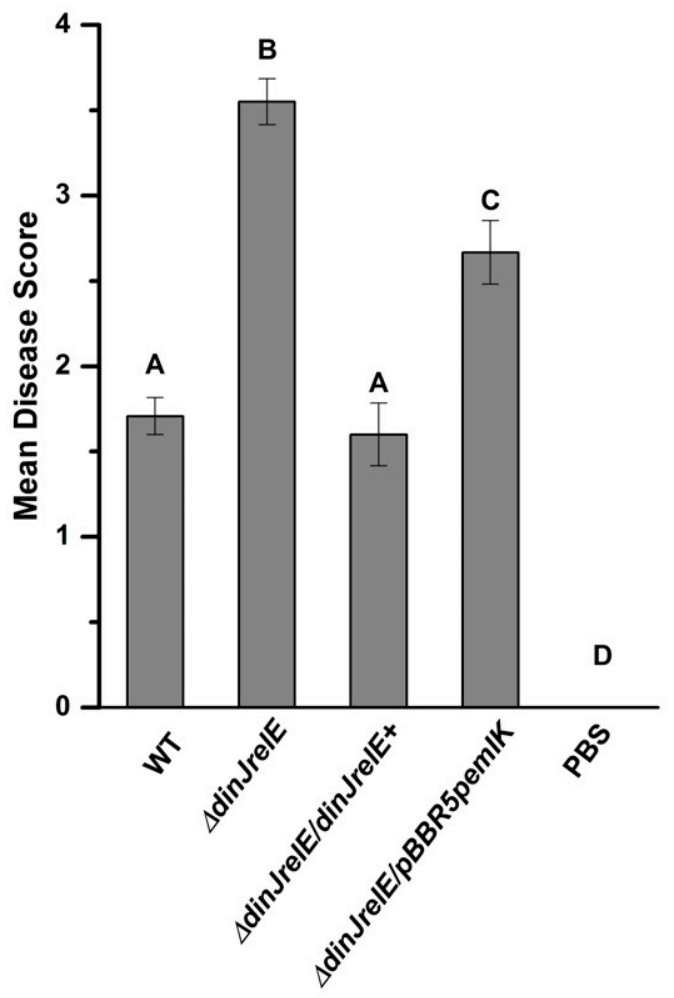

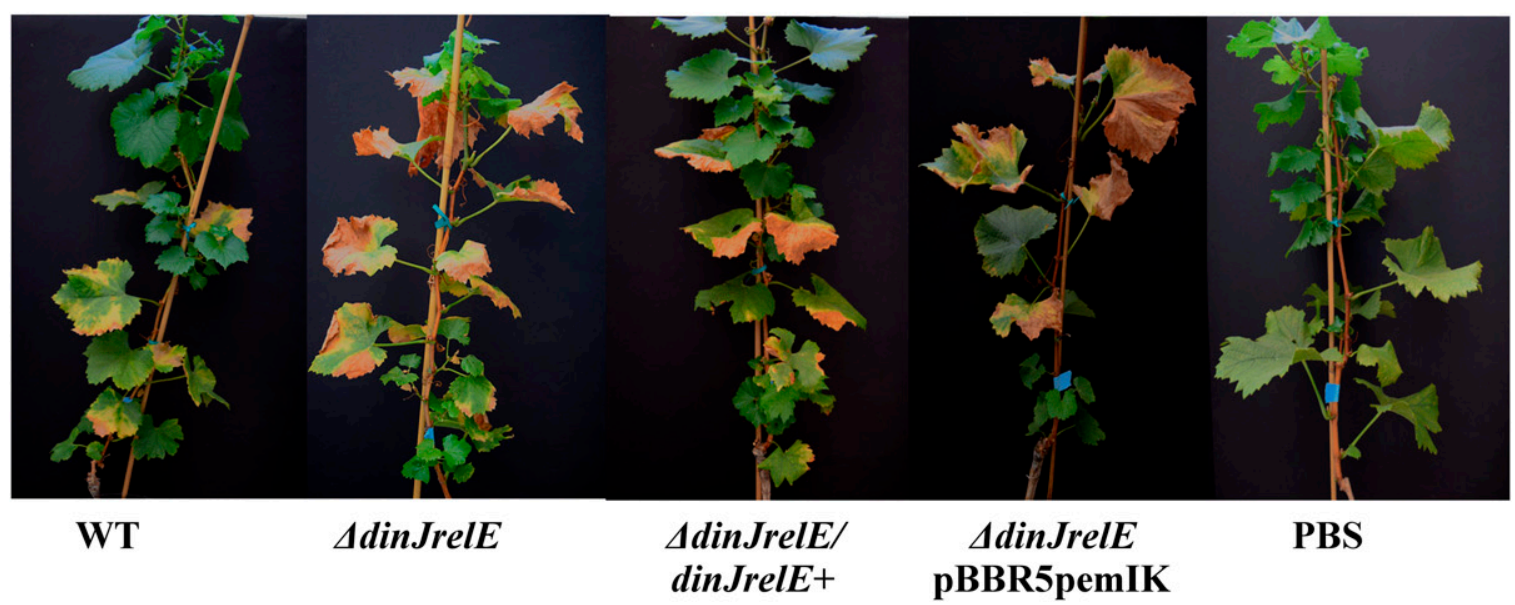

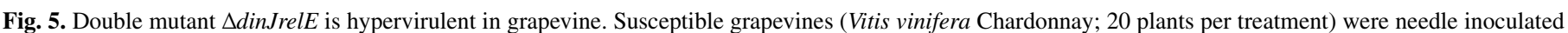

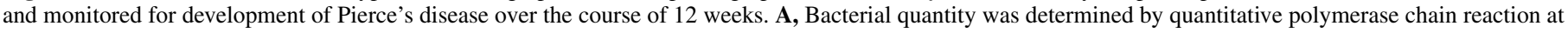

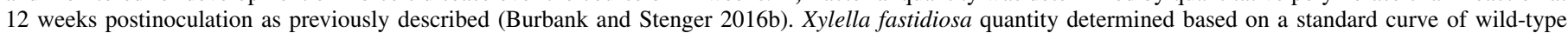

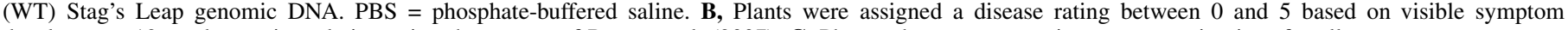
development 12 weeks postinoculation using the system of Roper et al. (2007). C, Photos show representative symptomatic vines for all treatments. 
We show that the DinJ/RelE TA system affects $X$. fastidiosa population growth in the plant. DinJ/RelE is encoded by two overlapping ORF located between bacteriophage gene sequences, suggesting acquisition by horizontal gene transfer (Lee et al. 2014). TA system toxins and cognate antitoxins are typically cotranscribed, with degradation of antitoxin proteins serving to regulate the amount of free toxin present in the cell. 5' RACE analysis of the dinJ/relE locus revealed the presence of two separate transcripts, demonstrating that the toxin and antitoxin are cotranscribed but that relE also can be expressed separately from an alternate promoter. Although cotranscription is the most common mechanism of bacterial TA system expression, presence of an internal promoter has been described for the Axe/Txe TA system in Enterococcus faecium (Boss et al. 2013; Chan and Espinosa 2016; Nieto et al. 2010; Ning et al. 2013). This additional level of gene regulation may allow for greater control of toxin-mediated growth arrest in response to different environmental stimuli. In many characterized TA systems, autoregulation through promoter binding of antitoxin or TA protein complexes also is an important factor in mediating TA system expression (Armalyte et al. 2012; Cataudella et al. 2012). Protein-binding interactions still need to be characterized for $X$. fastidiosa DinJ/RelE but it is possible that direct regulation of one or both of the promoters is a factor in this case as well. The ratio of dinJ:relE (antitoxin/toxin) expression at the transcriptional level was higher (3.7:1) during growth in rich medium (PD3) because of a sixfold increase in expression of $\operatorname{dinJ}$ compared with growth in Chard 2 medium. In Chard 2 medium, which is designed to mimic grapevine xylem sap, the antitoxin/toxin ratio was roughly $1: 1$. An antitoxin/toxin ratio of $1: 1$ at the transcriptional level would most likely correlate with higher amounts of free toxin in the cell, because antitoxin proteins are structurally unstable and more prone to proteolysis (Donegan et al. 2010; Loris et al. 2003; Nieto et al. 2007). Further characterization of RelE and DinJ proteins from $X$. fastisiosa would be necessary to fully understand stability dynamics in this specific case. Nonetheless, the transcriptional expression differences shown here demonstrate a response of the DinJ/RelE TA system to changes in the cellular growth environment. RelE homologs are often activated under starvation conditions to inhibit translation as part of a survival mechanism (Christensen et al. 2001). Due to the relatively limited nutrient content of xylem sap, it seems feasible that the $X$. fastidiosa DinJ/RelE TA system is used to reduce growth rate and cell density in planta, thereby facilitating long-term pathogen survival and preventing premature death of the host. Interestingly, the shift in antitoxin/toxin ratio in Chard2 appears to be a result of decreased expression from promoter $\mathrm{P} 1$ as compared with growth in rich medium. Expression from P2 appears to be constitutive and independent of growth medium, suggesting that a certain level of RelE is always present in the cell and that modulation of toxin activity based on growth conditions is mainly a result of changes in the amount of DinJ.

TA system activation is often associated with development of metabolically inactive persister cells which increase the long-term survival of the bacterial population due to limited nutrient requirements and increased resistance to stress (Fasani and Savageau 2015; Keren et al. 2004; Tashiro et al. 2012). It has previously been shown that a double mutant in $X$. fastidiosa $\Delta$ dinJ/relE has a higher proportion of viable cells during in vitro growth compared with the wild type. There is conflicting evidence, however, concerning whether or not the growth arrest and reduction in cell viability caused by TA system expression confers a long-term advantage on bacterial populations (Engelberg-Kulka et al. 2005; Pandey and Gerdes 2005; Pedersen et al. 2002; Tsilibaris et al. 2007). Although, after 7 days of growth, the $\Delta$ dinJrelE mutant exhibited a higher proportion of viable cells, at 14 days of growth there did not appear to be any significant difference between the TA system mutant and the wild type. Consequently, it does not appear that the DinJ/RelE TA system directly effects long-term survival in $X$. fastidiosa during in vitro growth.
There have been a number of reports of the importance of TA systems for virulence and microbe-host interactions in animal pathogens, although less evidence has been found for a role in plant-pathogen interactions (De la Cruz et al. 2013; LobatoMárquez et al. 2015; Wang et al. 2015). We found that, in the case of $X$. fastidiosa, the DinJ/RelE TA system suppresses virulence in grapevine, leading to a hypervirulent phenotype in the knockout mutant $\Delta$ dinJrelE. It is not out of the question that DinJ/RelE is involved in specific pathogen-host interactions in X. fastidiosa. In Xanthomonas oryzae, a pathogen of rice, there is at least one TA system that influences the host response to pathogen invasion through activity of the toxin as a type III secreted effector protein (Triplett et al. 2016). Although Xylella fastidiosa does not have a type III secretion system, there is some evidence that TA system proteins can be secreted by alternate mechanisms such as outer membrane vesicles (Merfa et al. 2016). However, in light of the observed changes in $\operatorname{din} J$ and relE expression in response to the different growth conditions compared, it seems likely that the suppression of virulence by DinJ/RelE is due to control of cell viability and proliferation in the host environment. Higher bacterial titer in $\Delta$ dinJrelE-inoculated plants would support this conclusion as well. Tempering the severity and rapidity of disease development through TA system activity could be advantageous to the pathogen by preventing death of the host and increasing the success of systemic spread and acquisition by the insect vector. The bacteriostatic effect of TA system expression also has been suggested as a mechanism for control of bacterial diseases (Engelberg-Kulka et al. 2004). Due to the observed hypervirulent phenotype of the $\Delta$ dinJrelE knockout mutant, there may be potential for suppression of Pierce's disease through artificial activation of this and other TA systems in $X$. fastidiosa.

\section{ACKNOWLEDGMENTS}

We thank K. Zhang and B. Ortega for technical support and M. S. Sisterson for assistance with statistical analysis. Funding for this work was from United States Department of Agriculture (USDA) Agricultural Research Service appropriated project 2034-22000-010-00D. Mention of trade names or commercial products in this publication is solely for the purpose of providing specific information and does not constitute endorsement by USDA. USDA is an equal opportunity provider and employer.

\section{LITERATURE CITED}

Armalytė, J., Jurènaitè, M., Beinoravičiūtè, G., Teišerskas, J., and Sužiedėlienė, E. 2012. Characterization of Escherichia coli dinJ-yafQ toxin-antitoxin system using insights from mutagenesis data. J. Bacteriol. 194:1523-1532.

Balaban, N. Q., Merrin, J., Chait, R., Kowalik, L., and Leibler, S. 2004. Bacterial persistence as a phenotypic switch. Science 305:1622-1625.

Boss, L., Labudda, Ł., Węgrzyn, G., Hayes, F., and Kędzierska, B. 2013. The Axe-Txe complex of Enterococcus faecium presents a multilayered mode of toxin-antitoxin gene expression regulation. PLoS One 8:e73569.

Burbank, L., Mohammadi, M., and Roper, M. C. 2015. Siderophore-mediated iron acquisition influences motility and is required for full virulence of the xylem-dwelling bacterial phytopathogen Pantoea stewartii subsp. stewartii. Appl. Environ. Microbiol. 81:139-148.

Burbank, L. P., and Stenger, D. C. 2016a. Plasmid vectors for Xylella fastidiosa utilizing a toxin-antitoxin system for stability in the absence of antibiotic selection. Phytopathology 106:928-936.

Burbank, L. P., and Stenger, D. C. 2016b. A temperature-independent coldshock protein homolog acts as a virulence factor in Xylella fastidiosa. Mol. Plant-Microbe Interact. 29:335-344.

Cataudella, I., Trusina, A., Sneppen, K., Gerdes, K., and Mitarai, N. 2012. Conditional cooperativity in toxin-antitoxin regulation prevents random toxin activation and promotes fast translational recovery. Nucleic Acids Res. 40:6424-6434.

Chan, W. T., and Espinosa, M. 2016. The Streptococcus pneumoniae pezAT toxin-antitoxin system reduces $\beta$-lactam resistance and genetic competence. Front. Microbiol. 7:1322.

Chatterjee, S., Almeida, R. P. P., and Lindow, S. 2008. Living in two worlds: The plant and insect lifestyles of Xylella fastidiosa. Annu. Rev. Phytopathol. 46:243-271. 
Chatterjee, S., Killiny, N., Almeida, R. P. P., and Lindow, S. E. 2010. Role of cyclic di-GMP in Xylella fastidiosa biofilm formation, plant virulence, and insect transmission. Mol. Plant-Microbe Interact. 23:1356-1363.

Chen, J., Wu, F., Zheng, Z., Deng, X., Burbank, L. P., and Stenger, D. C. 2016. Draft genome sequence of Xylella fastidiosa subsp. fastidiosa strain Stag's Leap. Genome Announc. 4:e00240-16.

Christensen, S. K., Mikkelsen, M., Pedersen, K., and Gerdes, K. 2001. RelE, a global inhibitor of translation, is activated during nutritional stress. Proc. Natl. Acad. Sci. USA 98:14328-14333.

Cook, G. M., Robson, J. R., Frampton, R. A., McKenzie, J., Przybilski, R., Fineran, P. C., and Arcus, V. L. 2013. Ribonucleases in bacterial toxinantitoxin systems. Biochim. Biophys. Acta 1829:523-531 (BBA).

Daugherty, M. P., Rashed, A., Almeida, R. P. P., and Perring, T. M. 2011. Vector preference for hosts differing in infection status: Sharpshooter movement and Xylella fastidiosa transmission. Ecol. Entomol. 36:654-662.

Davis, M., French, W., and Schaad, N. 1981. Axenic culture of the bacteria associated with phony disease of peach and plum leaf scald. Curr. Microbiol. 6:309-314.

Dehal, P. S., Joachimiak, M. P., Price, M. N., Bates, J. T., Baumohl, J. K., Chivian, D., Friedland, G. D., Huang, K. H., Keller, K., Novichkov, P. S., Dubchak, I. L., Alm, E. J., and Arkin, A. P. 2009. MicrobesOnline: An integrated portal for comparative and functional genomics. Nucleic Acids Res. 38:D396-D400.

De la Cruz, M. A., Zhao, W., Farenc, C., Gimenez, G., Raoult, D., Cambillau, C., Gorvel, J.-P., and Méresse, S. 2013. A toxin-antitoxin module of Salmonella promotes virulence in mice. PLoS Pathog. 9:e1003827.

Donegan, N. P., Thompson, E. T., Fu, Z., and Cheung, A. L. 2010. Proteolytic regulation of toxin-antitoxin systems by ClpPC in Staphylococcus aureus. J. Bacteriol. 192:1416-1422.

Engelberg-Kulka, H., Hazan, R., and Amitai, S. 2005. MazEF: Chromosomal toxin-antitoxin module that triggers programmed cell death in bacteria. J. Cell Sci. 118:4327-4332.

Engelberg-Kulka, H., Sat, B., Reches, M., Amitai, S., and Hazan, R. 2004. Bacterial programmed cell death systems as targets for antibiotics. Trends Microbiol. 12:66-71.

Fasani, R. A., and Savageau, M. A. 2015. Unrelated toxin-antitoxin systems cooperate to induce persistence. J. R. Soc. Interface 12:20150130.

Guilhabert, M. R., Hoffman, L. M., Mills, D. A., and Kirkpatrick, B. C. 2001. Transposon mutagenesis of Xylella fastidiosa by electroporation of Tn5 synaptic complexes. Mol. Plant-Microbe Interact. 14:701-706.

Heckman, K. L., and Pease, L. R. 2007. Gene splicing and mutagenesis by PCR-driven overlap extension. Nat. Protoc. 2:924-932.

Jahn, C. E., Charkowski, A. O., and Willis, D. K. 2008. Evaluation of isolation methods and RNA integrity for bacterial RNA quantitation. J. Microbiol. Methods 75:318-324.

Keren, I., Shah, D., Spoering, A., Kaldalu, N., and Lewis, K. 2004. Specialized persister cells and the mechanism of multidrug tolerance in Escherichia coli. J. Bacteriol. 186:8172-8180.

Killiny, N., and Almeida, R. P. P. 2009a. Xylella fastidiosa afimbrial adhesins mediate cell transmission to plants by leafhopper vectors. Appl. Environ. Microbiol. 75:521-528.

Killiny, N., and Almeida, R. P. P. 2009b. Host structural carbohydrate induces vector transmission of a bacterial plant pathogen. Proc. Natl. Acad. Sci. USA 106:22416-22420.

Kim, Y., Wang, X., Ma, Q., Zhang, X.-S., and Wood, T. K. 2009. Toxinantitoxin systems in Escherichia coli influence biofilm formation through YjgK (TabA) and fimbriae. J. Bacteriol. 191:1258-1267.

Korch, S. B., Contreras, H., and Clark-Curtiss, J. E. 2009. Three Mycobacterium tuberculosis Rel toxin-antitoxin modules inhibit mycobacterial growth and are expressed in infected human macrophages. J. Bacteriol. 191: 1618-1630.

Ledbetter, C. A., and Rogers, E. E. 2009. Differential susceptibility of Prunus germplasm (subgenus Amygdalus) to a California isolate of Xylella fastidiosa. HortScience 44:1928-1931.

Lee, M. W., Rogers, E. E., and Stenger, D. C. 2012. Xylella fastidiosa plasmidencoded PemK toxin is an endoribonuclease. Phytopathology 102:32-40.

Lee, M. W., Tan, C. C., Rogers, E. E., and Stenger, D. C. 2014. Toxin-antitoxin systems mqsR/ygiT and dinJ/relE of Xylella fastidiosa. Physiol. Mol. Plant Pathol. 87:59-68.

Leite, B., Andersen, P. C., and Ishida, M. L. 2004. Colony aggregation and biofilm formation in xylem chemistry-based media for Xylella fastidiosa. FEMS Microbiol. Lett. 230:283-290.

Livak, K. J., and Schmittgen, T. D. 2001. Analysis of relative gene expression data using real-time quantitative PCR and the $2^{-\Delta \Delta C T}$ method. Methods 25: 402-408.

Lobato-Márquez, D., Díaz-Orejas, R., and García-del Portillo, F. 2016. Toxinantitoxin systems and bacterial virulence. FEMS Microbiol. Rev. 40: 592-609.
Lobato-Márquez, D., Moreno-Córdoba, I., Figueroa, V., Díaz-Orejas, R., and García-del Portillo, F. 2015. Distinct type I and type II toxin-antitoxin modules control Salmonella lifestyle inside eukaryotic cells. Sci. Rep. 5: Article number 9374. doi:10.1038/srep09374

Loris, R., Marianovsky, I., Lah, J., Laeremans, T., Engelberg-Kulka, H., Glaser, G., Muyldermans, S., and Wyns, L. 2003. Crystal structure of the intrinsically flexible addiction antidote MazE. J. Biol. Chem. 278:2825228257.

Martins, P. M. M., Machado, M. A., Silva, N. V., Takita, M. A., and de Souza, A. A. 2016. Type II toxin-antitoxin distribution and adaptive aspects on Xanthomonas genomes: Focus on Xanthomonas citri. Front. Microbiol. 7: 652.

Matsumoto, A., Young, G. M., and Igo, M. M. 2009. Chromosome-based genetic complementation system for Xylella fastidiosa. Appl. Environ. Microbiol. 75:1679-1687.

Merfa, M. V., Niza, B., Takita, M. A., and De Souza, A. A. 2016. The MqsRA toxin-antitoxin system from Xylella fastidiosa plays a key role in bacterial fitness, pathogenicity, and persister cell formation. Front. Microbiol. 7:904.

Minsavage, G. V., Thomson, C. M., Hopkins, D. L., Leite, R. M. V. B. C., and Stall, R. E. 1994. Development of a polymerase chain reaction protocol for detection of Xylella fastidiosa in plant tissue. Phytopathology 84:456-461.

Newman, K. L., Almeida, R. P. P., Purcell, A. H., and Lindow, S. E. 2003. Use of a green fluorescent strain for analysis of Xylella fastidiosa colonization of Vitis vinifera. Appl. Environ. Microbiol. 69:7319-7327.

Newman, K. L., Almeida, R. P. P., Purcell, A. H., and Lindow, S. E. 2004. Cell-cell signaling controls Xylella fastidiosa interactions with both insects and plants. Proc. Natl. Acad. Sci. USA 101:1737-1742.

Nieto, C., Cherny, I., Khoo, S. K., de Lacoba, M. G., Chan, W. T., Yeo, C. C., Gazit, E., and Espinosa, M. 2007. The yefM-yoeB toxin-antitoxin systems of Escherichia coli and Streptococcus pneumoniae: Functional and structural correlation. J. Bacteriol. 189:1266-1278.

Nieto, C., Sadowy, E., de la Campa, A. G., Hryniewicz, W., and Espinosa, M. 2010. The relBESpn toxin-antitoxin system of Streptococcus pneumoniae: Role in antibiotic tolerance and functional conservation in clinical isolates. PLoS One 5:e11289.

Ning, D., Liu, S., Xu, W., Zhuang, Q., Wen, C., and Tang, X. 2013. Transcriptional and proteolytic regulation of the toxin-antitoxin locus vapBC10 (ssr2962/slr1767) on the chromosome of Synechocystis sp. PCC 6803. PLoS One 8:e80716.

Pandey, D. P., and Gerdes, K. 2005. Toxin-antitoxin loci are highly abundant in free-living but lost from host-associated prokaryotes. Nucleic Acids Res. 33:966-976.

Pedersen, K., Christensen, S. K., and Gerdes, K. 2002. Rapid induction and reversal of a bacteriostatic condition by controlled expression of toxins and antitoxins. Mol. Microbiol. 45:501-510.

Roper, M. C., Greve, L. C., Warren, J. G., Labavitch, J. M., and Kirkpatrick, B. C. 2007. Xylella fastidiosa requires polygalacturonase for colonization and pathogenicity in Vitis vinifera grapevines. Mol. Plant-Microbe Interact. 20:411-419.

Scotto-Lavino, E., Du, G., and Frohman, M. A. 2007. 5' end cDNA amplification using classic RACE. Nat. Protoc. 1:2555-2562.

Shiloh, M. U., Ruan, J., and Nathan, C. 1997. Evaluation of bacterial survival and phagocyte function with a fluorescence-based microplate assay. Infect. Immun. 65:3193-3198.

Syed, M. A., and Lévesque, C. M. 2012. Chromosomal bacterial type II toxin-antitoxin systems. Can. J. Microbiol. 58:553-562.

Tashiro, Y., Kawata, K., Taniuchi, A., Kakinuma, K., May, T., and Okabe, S. 2012. RelE-mediated dormancy is enhanced at high cell density in Escherichia coli. J. Bacteriol. 194:1169-1176.

Triplett, L. R., Shidore, T., Long, J., Miao, J., Wu, S., Han, Q., Zhou, C., Ishihara, H., Li, J., Zhao, B., and Leach, J. E. 2016. AvrRxo1 is a bifunctional type III secreted effector and toxin-antitoxin system component with homologs in diverse environmental contexts. PLoS One 11:e0158856.

Tsilibaris, V., Maenhaut-Michel, G., Mine, N., and Van Melderen, L. 2007. What is the benefit to Escherichia coli of having multiple toxin-antitoxin systems in its genome? J. Bacteriol. 189:6101-6108.

Wang, N., Li, J.-L., and Lindow, S. E. 2012. RpfF-dependent regulon of Xylella fastidiosa. Phytopathology 102:1045-1053.

Wang, Y., Wang, H., Hay, A. J., Zhong, Z., Zhu, J., and Kan, B. 2015. Functional RelBE-family toxin-antitoxin pairs affect biofilm maturation and intestine colonization in Vibrio cholerae. PLoS One 10:e135696.

Yamaguchi, Y., and Inouye, M. 2011. Regulation of growth and death in Escherichia coli by toxin-antitoxin systems. Nat. Rev. Microbiol. 9:779790

Zaini, P. A., Fogaça, A. C., Lupo, F. G. N., Nakaya, H. I., Vêncio, R. Z. N., and da Silva, A. M. 2008. The iron stimulon of Xylella fastidiosa includes genes for type IV pilus and colicin V-like bacteriocins. J. Bacteriol. 190: 2368-2378. 